\title{
Heart Dysfunction Following Long-Term Murine Cytomegalovirus Infection: Fibrosis, Hypertrophy, and Tachycardia
}

\author{
Cassandra M. Bonavita, ${ }^{1}$ Timothy M. White, ${ }^{1}$ Joseph Francis, ${ }^{2}$ and Rhonda D. Cardin ${ }^{1}$
}

\begin{abstract}
Human cytomegalovirus (HCMV) is associated with increased risk of chronic diseases of the heart and vasculature, including myocarditis, atherosclerosis, and transplant vasculopathy. To investigate CMV infection of the heart, murine cytomegalovirus (MCMV) was used to evaluate both acute and latent infection and the subsequent phenotypic and functional consequences of infection. Female BALB/c mice were intraperitoneally (i.p.) inoculated with $1 \times 10^{6} \mathrm{pfu}$ of MCMV and evaluated at 14 and 50 days postinfection (dpi). At each time point, echocardiography was used to evaluate cardiac function and histology was conducted for phenotypic evaluation. MCMV replication in the heart was detected as early as 3 dpi and was no longer detectable at 14 dpi. Infected animals had significant cardiac pathology at 14 and 50 dpi when compared to uninfected controls. Histology revealed fibrosis of the heart as early as $14 \mathrm{dpi}$ and the presence of white fibrous deposits on the surface of the heart. Functional evaluation showed significantly increased heart rate and muscle thickening in the latently infected animals when compared to the control animals. At $50 \mathrm{dpi}$, latent virus was measured by explant reactivation assay, demonstrating that MCMV establishes latency and is capable of reactivation from the heart, similar to other tissues such as spleen and salivary glands. Collectively, these studies illustrate that MCMV infection results in phenotypic alterations within the heart as early as $14 \mathrm{dpi}$, which progress to functional abnormalities during latency. These findings are similar to sinus tachycardia and hypertrophy of the heart muscle observed in cases of HCMV-induced acute myocarditis.
\end{abstract}

Keywords: cytomegalovirus, murine cytomegalovirus, heart function, myocarditis, viral pathogenesis, viral reactivation

\section{Introduction}

$\mathbf{H}$ UMAN CYTOMEGALOVIRUS (HCMV), a beta-herpesvirus, is a ubiquitous pathogen that infects $50-90 \%$ of the world population and results in lifelong latent infection characterized by frequent reactivations. While infection is generally asymptomatic in immunocompetent populations, increasing evidence shows a correlation between HCMV seropositivity and chronic diseases (34). HCMV has long been associated with cardiovascular complications (17). HCMVassociated cardiovascular dysfunction includes increased morbidity during atherosclerosis, myocarditis, and transplant vasculopathy $(20,32,34,41)$. Myocarditis is defined as inflammation of the heart muscle caused by infiltrating immune cells, which results in dysfunction of the heart. While HCMV- induced myocarditis is rare in immunocompetent adults, acute myocarditis can occur and is life-threatening if untreated (40).

In HCMV-seropositive individuals, studies have identified viral DNA and protein expression within the heart as well as detectable serum IgM and IgG (31). Acute HCMV infection can result in myocarditis with symptoms of sinus tachycardia, ventricular overload, and hypertrophy $(18,24,27)$. Administration of the HCMV antiviral drug valganciclovir reduces complications associated with infection and returns the heart to normal function within 1 month of treatment (27). A related beta-herpesvirus, human herpesvirus-6 (HHV-6), infects endothelial cells of the aorta and heart, increasing their proinflammatory secretions as well as contributing to complications following heart transplant $(5,15,16,19)$.

Departments of ${ }^{1}$ Pathobiological Sciences and ${ }^{2}$ Comparative Biological Sciences, School of Veterinary Medicine, Louisiana State University, Baton Rouge, Louisiana.

(C) Cassandra M. Bonavita et al., 2020; Published by Mary Ann Liebert, Inc. This Open Access article is distributed under the terms of the Creative Commons Attribution Noncommercial License (http://creativecommons.org/licenses/by-nc/4.0/) which permits any noncommercial use, distribution, and reproduction in any medium, provided the original author(s) and the source are cited. 
Due to the life-threatening complications of acute HCMV infection in the heart and association with chronic cardiovascular dysfunction, understanding the viral mechanisms at play within the heart is crucial. Murine cytomegalovirus (MCMV) shares close homology with HCMV and recapitulates most characteristics of infection (11). As such, MCMV can be used to model HCMV pathogenesis of the heart and vasculature $(6,21,22,28,30,38,39)$. Previous studies have shown that MCMV replication in the heart peaks at 6 days postinfection (dpi) and clears within 14 days; however, viral DNA has been detected as late as $100 \mathrm{dpi}$ (21).

MCMV infection of the heart leads to $\mathrm{CD}^{+} \mathrm{T}$ cell infiltration at early times and expression of proinflammatory cytokines, including interferon gamma (IFN $\gamma$ ), interleukin-6 (IL-6), and tumor necrosis factor alpha (TNF $\alpha)(30)$. Infection is not isolated to a single region of the heart, but is found sporadically in the entire tissue (30). Ganciclovir and cidofovir treatment reduces myocarditis during MCMV infection, similar to HCMV (22). In addition to modeling HCMV-induced myocarditis, MCMV exacerbates atherosclerotic plaque size and plaque instability in APOE knockout mice $(6,38,39)$. Transplant vasculopathy from transplantation of a latent MCMV-infected heart allograft also results in widespread fibrosis and increased rejection of the organ, similar to $\operatorname{HCMV}(8,35)$.

Previous MCMV studies have been used to determine the cell types infected, the response of the immune system, and cytokines that contribute to inflammation in the heart $(6,21,22,28,30,38,39)$. However, to the best of our knowledge, this is the first study that has evaluated functional changes in the heart following MCMV infection, which show similarities to HCMV-induced myocarditis. We hypothesized that MCMV infection of the heart would result in acute phenotypic changes that could potentially lead to heart dysfunction. In this study, we use echocardiography to measure the heart rate, wall motion abnormalities (heartbeat), and histological changes in the heart following MCMV infection. Interestingly, our results indicate that persistent MCMV infection of the heart may lead to continued dysfunction.

\section{Materials and Methods}

\section{Cell culture conditions and virus preparation}

NIH 3 T3 cells (Cell Line Services, Eppelheim, Germany) were grown in Dulbecco's modified Eagle's medium (DMEM) with $4.5 \mathrm{~g} / \mathrm{L}$ glucose and L-glutamine without sodium pyruvate (Corning, NY), supplemented with $10 \%$ fetal bovine serum (HyClone), $7.5 \%$ sodium bicarbonate, $4 \mathrm{mM}$ HEPES, and $2 \mathrm{mM}$ L-glutamine, and $1 \%$ gentamicin. Cells were incubated in $5 \% \mathrm{CO}_{2}$ at $37^{\circ} \mathrm{C}$. Stocks of MCMV strains, K181+ Stanford and RM427+, were originally obtained from Edward Mocarski (35), and K181 Perth (KP) was provided by Hellen Farrell (4). Virus stocks were prepared from salivary gland-derived stocks in NIH $3 \mathrm{~T} 3$ fibroblasts, as previously described (9). The virus stock titer was determined by plaque assay and stocks were stored at $-80^{\circ} \mathrm{C}$.

\section{Mice and infection}

Five-week-old female BALB/c mice were purchased from Jackson Laboratory, Bar Harbor. For MCMV infection, mice were uninfected or intraperitoneally (i.p.) inoculated with $1 \times 10^{6}$ pfu of the MCMV strain KP unless otherwise stated. At 3, 7, 14, and $50 \mathrm{dpi}$, animals were sacrificed and evaluated. Mice were maintained under specific pathogen-free conditions at Louisiana State University School of Veterinary Medicine, Baton Rouge, LA. All animal protocols were approved by the Institutional Animal Care and Use Committee at Louisiana State University.

\section{Virus replication}

Virus replication in the hearts, spleens, livers, lungs, and salivary glands from infected mice was evaluated by plaque assay at 3, 7, and 14 dpi. Hearts were weighed and $10 \%$ homogenates (w/v) were prepared and sonicated (1). Tissue samples were titered by plaque assay in NIH 3T3 cells. After incubation at $37^{\circ} \mathrm{C}$ in $5 \% \mathrm{CO}_{2}$, monolayers were stained with Giemsa stain and plaques were counted. Virus titers are represented as $\log _{10} \mathrm{PFU} / \mathrm{mL}$. Group mean and standard error of the mean were calculated and significance was determined by Student's $t$ test. Studies were conducted in duplicate $(n=10)$ (GraphPad, San Diego, CA).

\section{Quantitative polymerase chain reaction}

The presence of viral DNA within the hearts was evaluated by quantitative polymerase chain reaction (qPCR). From each heart, $0.2 \mathrm{~mL}$ of tissue homogenate was collected and DNA was isolated using the QIAamp DNA extraction kit (Qiagen, Hilden, Germany). The E1 gene was amplified using E1-specific primers, (F-5'TCGCCCATCGTTTCGAGA-3') and (R-5'TCT CGTAGGTCCACTGACGGA-3'), and a probe (6FAM-ACT CGAGTCGGACGCTGCATCAGAAT-TAMRA), which amplified a 106-bp fragment (1). DNA samples were diluted to the lowest concentration sample ( $\sim 180 \mathrm{ng})$, and PCR was conducted with Promega master mix (Promega, Madison, WI), $1 \mathrm{nmol}$ of primers, and 2 pmol of probe in a $10-\mu \mathrm{L}$ volume. MCMV-infected NIH 3T3 cells were used as a positive control as well as a minus template control. qPCR was conducted using a AB7000 machine (Applied Biosystems, Foster City, CA) with the temperature profile of $50^{\circ} \mathrm{C}$ for $2 \mathrm{~min}, 95^{\circ} \mathrm{C}$ for $10 \mathrm{~min}$, and 40 cycles at $95^{\circ} \mathrm{C}$ for $15 \mathrm{sec}, 60^{\circ} \mathrm{C}$ for $1 \mathrm{~min}$, and $70^{\circ} \mathrm{C}$ for $1 \mathrm{~min}$. Viral DNA was visualized by electrophoresis on a $2 \%$ agarose gel.

\section{Pathological score}

Hearts were collected, washed with phosphate-buffered saline (PBS), and photographed to document the presence of white deposits. Pathology scores were determined based on the estimated percentage of white deposits visible on the surface of the heart: (0) no visible changes to the heart; $(0.5)$ hearts with no visible white deposit, but altered pale appearance; (1) $0-25 \%$ of the heart containing white deposit; (2) $25-50 \%$ of the heart containing white deposit; (3) $50-$ $75 \%$ of the heart containing white deposit; and (4) 75-100\% of the heart containing white deposit. White deposits were assessed on both the front and back of the heart. Scores were averaged, standard error of the mean was calculated, and significance was determined by the Mann-Whitney $U$ test (GraphPad).

\section{Echocardiography}

At 14 and $50 \mathrm{dpi}$, uninfected or MCMV-infected mice were evaluated for functional alterations of the heart by 
echocardiography (Toshiba Aplio 80, Tokyo, Japan). Mice were sedated with $2 \%$ isoflurane inhalation through a nose cone, their chest walls were shaved, covered in transmission gel, and echocardiography was conducted on the anterior chest wall (9). Heart rate and wall motion abnormalities were assessed using the following parameters: left ventricular internal diameter diastolic/systolic, left ventricular posterior wall thickness diastolic/systolic, end-diastolic/systolic volume, stroke volume, ejection fraction, and fractional shortening. Group means and standard errors of the mean were calculated and Student's $t$ test was used to determine significance. Studies were conducted in duplicate at $14 \mathrm{dpi}$ $(n=10)$ and in triplicate at $50 \mathrm{dpi}(n=15)$.

\section{Histology}

At 14 and $50 \mathrm{dpi}$, mice were evaluated for phenotypic changes of the heart. Two hearts from each group analyzed by echocardiography were fixed in $10 \%$ formalin and histology was performed. Hearts were stained with Masson's trichrome to assess fibrotic changes and collagen accumulation and haemotoxylin and eosin (H\&E) to evaluate immune cell infiltration. Images where analyzed with NanoZoomer Digital Pathology 2 software. Studies were conducted in triplicate $(n=6)$.

\section{Latency evaluation}

At 50 dpi, mice were evaluated for viral reactivation using a primary explant reactivation assay, as previously described (1). Briefly, three hearts and spleens were collected following echocardiography, minced, and cultured in DMEM for 6 weeks. Every 7 days, the supernatant was collected and infectious virus was evaluated by plaque assay to determine reactivation from latency. Latency within the tissues is defined by the absence of infectious virus in the tissue at the time of explant and at 7 days post explant (dpe). Plaques detected after 7 dpe indicate infectious virus due to reactivation of MCMV within the cultured tissue. The spleen is a well-characterized tissue for this assay and was included as a viral reactivation control. Studies were conducted in triplicate $(n=9)$ and graphed as the percent of animals reactivating from latency.

\section{Statistics}

All results were analyzed using group mean, standard error of the mean, and Student's $t$ test or Mann-Whitney $U$ test. Statistics and figures were produced using Prism 8 software. Significance was set as $p=0.01 *, 0.001^{* *}, 0.0001^{* * *}$, and $<0.00001 * * * *$ (GraphPad).

\section{Results}

\section{Infection of the heart and replication kinetics}

Infection of the heart was assessed following i.p. inoculation with $1 \times 10^{6} \mathrm{pfu}$ of MCMV, KP strain. At 3, 7, and $14 \mathrm{dpi}$, heart homogenates were analyzed by plaque assay. Figure 1 shows MCMV infection kinetics of the heart. Similar to previous studies, infectious virus was detected at $3 \mathrm{dpi}$, but at $14 \mathrm{dpi}$, it was below the limit of detection by plaque assay $(21,30)$. Infection of the heart resulted in a peak titer of $\sim 1 \log _{10} \mathrm{PFU} / \mathrm{mL}$ at $7 \mathrm{dpi}$, much lower compared with other tissues such as the spleen, liver, lung, and salivary gland. In addition to evaluating the infectious virus

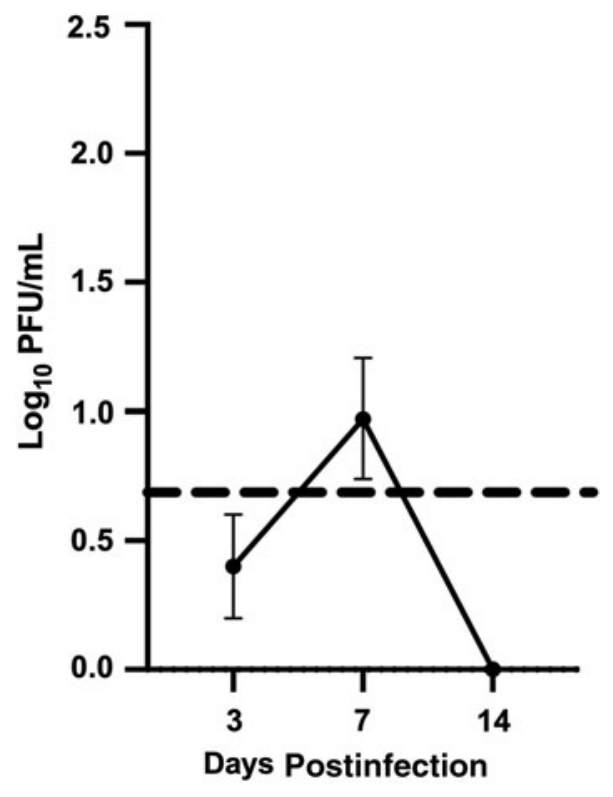

FIG. 1. MCMV infection of the heart. Mice were MCMV infected with $1 \times 10^{6}$ pfu i.p. At 3, 7, and 14 dpi, hearts were collected and analyzed by plaque assay. The dotted line in this figure is the limit of detection of the plaque assay. The study was conducted in duplicate, $n=8$. Group mean and standard error of the mean were calculated and significance was assessed by Student's $t$ test $(p=n s)$. dpi, days postinfection; i.p., intraperitoneally; MCMV, murine cytomegalovirus; ns, not significant.

by plaque assay, viral DNA levels in the heart were assessed by qPCR. The presence of viral DNA was visible at all time points in the MCMV-infected animals, with peak levels at $7 \mathrm{dpi}$ and reduced viral DNA detected at $14 \mathrm{dpi}$ (data not shown). To ensure there was no strain variability, the MCMV strain K181+ was also evaluated and results were similar at each time point (data not shown). These results are in agreement with previous results $(21,30)$.

\section{Phenotypic alterations of the heart}

The presence of white deposits on the pericardium of the heart following MCMV infection has previously been reported (30). Similarly, in our studies, white deposits were detected on hearts following inoculation with $1 \times 10^{6} \mathrm{pfu}$ of $\mathrm{KP}$ as early as $3 \mathrm{dpi}$. As shown in Figure 2A, white deposits were generally first observed in the right atrial region, followed by development at additional locations across the pericardium. Some deposits were heavily concentrated in one region, whereas other deposits occurred as small isolated spots across the surface of the heart. In rare instances, small deposits were observed on uninfected hearts; however, MCMV infection greatly exacerbated this phenotype. To determine whether white deposits were transient and resolve after active virus replication or persist into latent infection, mice were inoculated i.p. with $1 \times 10^{6}$ pfu of KP and sacrificed at 14 and 50 dpi. Hearts were washed in PBS and photographed to assess pathology using a score (0 to 4) estimating the percentage of the heart that contained white deposits. Figure 2B shows a significant increase in white deposits on infected hearts compared with uninfected 

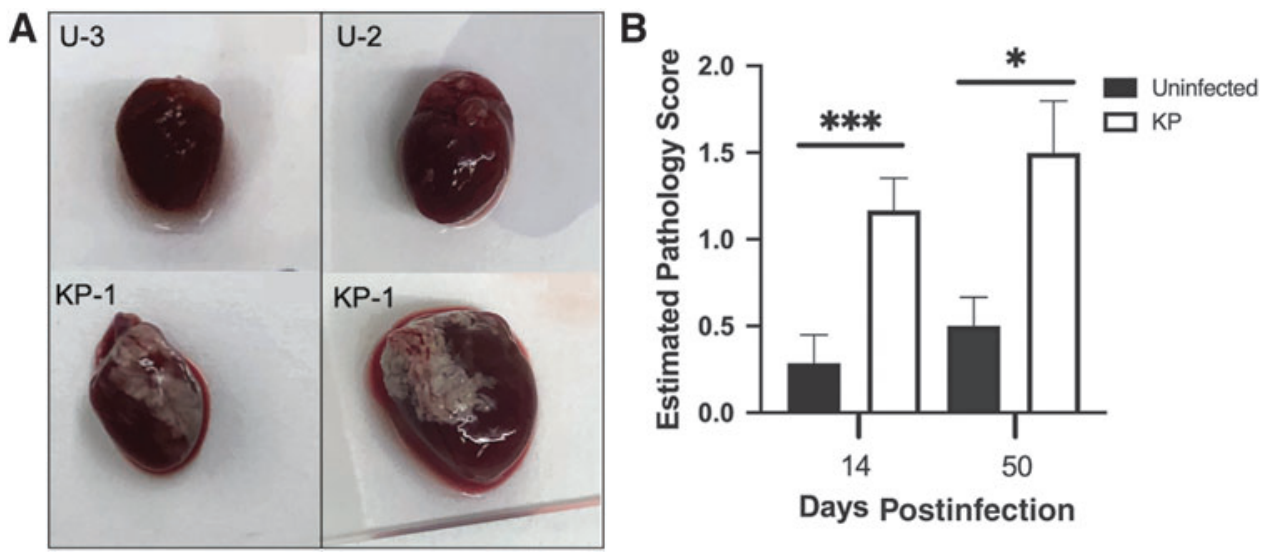

FIG. 2. White fibrous deposits on the surface of the pericardium. (A) Photographs of white deposits at 14 and 50 dpi from representative uninfected and MCMV-infected hearts. (B) Hearts were assessed for white deposits at 14 and 50 dpi and assigned a pathology score based on the percentage of white deposits on the pericardium. Studies were conducted in duplicate at $14 \mathrm{dpi}(n=10)$ and in triplicate at $50 \mathrm{dpi}(n=15)$. Group mean and standard error of the mean were calculated and significance was determined by the Mann-Whitney $U$ test $(* * * p=0.0001$ and $* p=0.01)$.

controls. Surprisingly, the average score in infected animals was similar at both time points, although a slight increase in both uninfected and KP-infected mice was detected at 50 dpi (not significant). To ensure this was not a strain-specific effect, mice were infected with the K181+ and RM427+ viruses and similar results were observed (data not shown). In addition, the inoculum dose was reduced to $1 \times 10^{5} \mathrm{pfu}$ of MCMV and white deposit formation was still observed, although at a lower score and frequency (data not shown). This result suggests that the deposits correlate with infection, although the white deposits persist until $50 \mathrm{dpi}$, a time when MCMV is latent in BALB/c mice (1), suggesting that active infection may not be required to maintain the presence of white deposits on the pericardium.

Next, phenotypic changes within the heart were evaluated by histology. At each time point, hearts were washed with PBS, formalin-fixed upon collection, embedded in paraffin, sectioned, and stained with Masson's trichrome. Masson's trichrome is used to assess fibrosis, as shown by blue staining of collagen accumulation within the tissue. Figure 3 shows the accumulation of collagen in MCMV-infected animals over time. At 14 dpi (Fig. 3A), uninfected hearts have little to no blue staining, while infected animals ex- hibited interstitial fibrosis in the epicardium of the heart located where the white deposits were observed (36). By 50 dpi (Fig. 3B), substantially increased fibrosis was detected throughout the inner layers of infected hearts, unlike uninfected control animals that exhibited very little collagen accumulation. In addition, the interstitial fibrosis visible within the heart at $14 \mathrm{dpi}$ had progressed to replacement fibrosis at $50 \mathrm{dpi}$, suggesting persistent damage within the heart tissue (36). Hearts were also stained with $\mathrm{H} \& \mathrm{E}$ to evaluate immune cell infiltration; however, results showed no detectable infiltration at 14 and $50 \mathrm{dpi}$, although it has been reported at 5-7 dpi (30). This result agrees with previous studies of MCMV-induced fibrosis, including allograft transplant hearts, which exhibit fibrosis 45 days after transplantation $(30,35)$. In addition, several in vitro studies demonstrate HCMV induction of profibrotic factors $(3,7,10,25,29,37)$.

\section{Heart function studies}

Based on the phenotypic alteration detected within the infected hearts, we hypothesized that the acute phenotypic changes in the heart may be maintained during latency and could result in long-term functional deficiencies. To assess
A

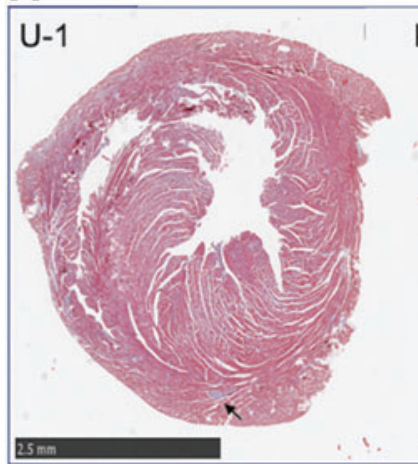

B

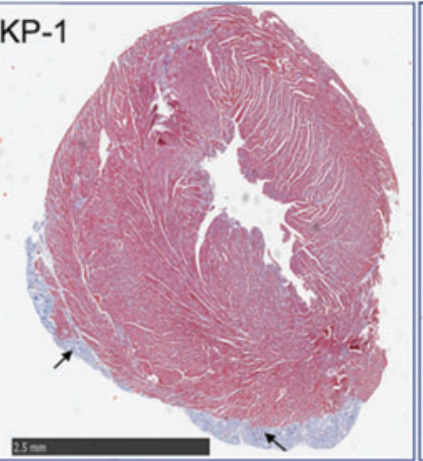

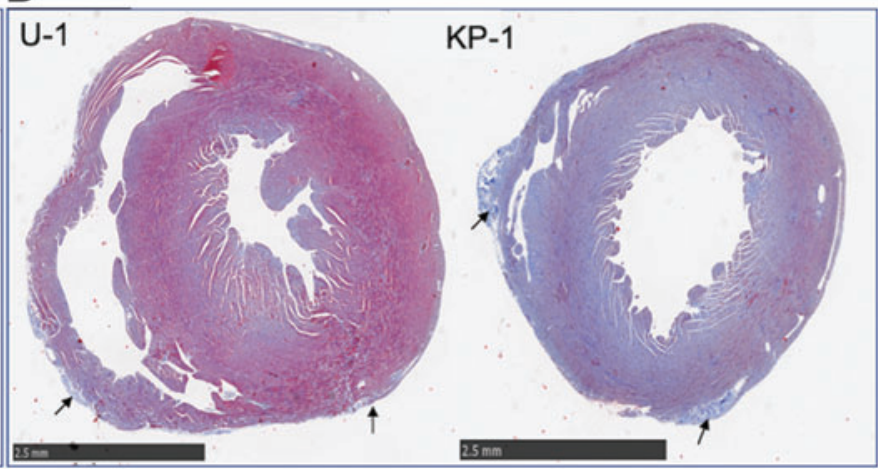

FIG. 3. Histology of the heart. Uninfected and MCMV-infected hearts at (A) 14 dpi and (B) 50 dpi were stained with Masson's trichrome to detect areas of collagen accumulation (blue staining and arrows). Images are representative of studies conducted in triplicate with $n=6$. 
this hypothesis, echocardiography was performed to evaluate heart function at 14 and 50 dpi. Echocardiography uses ultrasound waves to visualize the heart in real time and allows the assessment of functional features such as heart rate, muscle thickness, and wall motion abnormalities. Figure 4 shows that MCMV-infected mice have significantly higher heart rates than the uninfected control mice at both 14 and $50 \mathrm{dpi}, \sim 437$ and $\sim 447$ beats per minute, respectively, whereas the uninfected control mice exhibit a consistent heart rate of approximately $350-375$ beats per minute at both time points. Despite the increased fibrosis at $50 \mathrm{dpi}$, the heart rate does not differ between time points in MCMVinfected hearts, suggesting that perhaps the initial damage caused by infection is enough to maintain the heart rate abnormality. This result mimics sinus tachycardia seen in humans during HCMV-induced myocarditis, which requires valganciclovir treatment to return the heart to normal function $(18,24,27)$. These similarities between HCMV and MCMV further suggest that the murine model of cardiovascular dysfunction could reveal HCMV-associated cardiovascular complications.

In addition to the heart rate, other functional parameters of the heart were evaluated, including left ventricle internal diameter, left ventricle posterior wall thickness, end-diastolic volume, stroke volume, ejection fraction, and fraction shortening. Left ventricular internal diameter and left ventricular posterior wall thickness are measurements of the chambers and walls of the left ventricle during the systolic and diastolic phases of beating, which are used to determine muscle thickness and chamber capacity. End-systolic and diastolic loads are measurements of the volume of blood inside the left ventricle at the end of each phase and how much blood is ejected during each heartbeat and are used to determine cardiac output. Ejection fraction and fraction shortening measure heart contractibility and are used as metrics for cardiac function evaluation in humans (14). All of these metrics together are used in human and animal medicine to evaluate heart performance and overall health. These functional parameters at 14 and 50 dpi are shown in Figure 5.

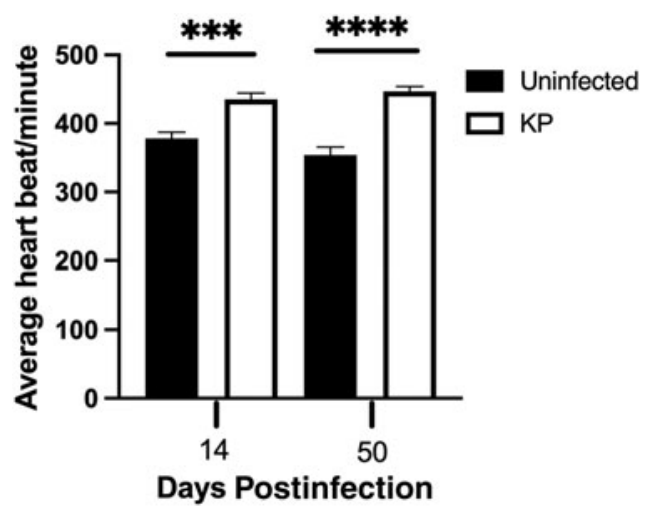

FIG. 4. Heart rate of uninfected and MCMV-infected mice at 14 and 50 dpi. The heart rate was assessed by echocardiography. The group mean and standard error of the mean were calculated and significance was determined by Student's $t$ test, $* * * p=0.0001$ and $* * * * p \leq 0.00001$. Studies were conducted in duplicate at $14 \mathrm{dpi},(n=10)$ and in triplicate at $50 \mathrm{dpi}(n=15)$.
Unlike the heart rate, there were no significant differences in any of these parameters at $14 \mathrm{dpi}$. However, at $50 \mathrm{dpi}$, Figure 5B shows that the left ventricular posterior wall thickness was significantly increased in both diastolic and systolic phases in MCMV-infected animals when compared with uninfected control mice. This result suggests hypertrophy of the heart muscle at 50 dpi and likely resulted from the fibrosis shown in Figure 3. Increased fibrosis within the tissue most likely requires the muscle to increase to function as efficiently as before the damage occurred. In addition to hypertrophy, Figure 5A shows a slight decrease in the left ventricular internal diameter during the diastolic phase, suggesting that less blood is ejected out of the ventricle during each systole. This may also be due to the fibrosis in the heart. Significantly, when visualizing the rhythm of contraction, the hearts of MCMV-infected mice had a slight spasm during each beat compared with uninfected control hearts. This suggests that electrical contraction signals may have a slight delay due to fibrosis within the heart at 50 dpi. Previous studies have shown that viral infection of the heart can cause abnormal ventricular ejection in humans (23). The remaining parameters were not affected in these mice at either time point, which might be expected since the infected mice did not exhibit overt signs of distress or heart failure. Together, Figures 4 and 5 demonstrate functional changes in the heart at 14 and $50 \mathrm{dpi}$, with more significant changes during latent infection.

\section{Ex vivo reactivation study}

As our data show, continued cardiac damage and functional abnormalities persist long after systemic MCMV replication has ended (1). Therefore, latency status and capacity for virus reactivation were evaluated in hearts and spleens of infected mice, which were cultured ex vivo by the explant reactivation assay (4). Reactivation of MCMV from salivary glands, lungs, and liver has been characterized by our laboratory previously (1). However, to the best of our knowledge, this is the first study to evaluate virus reactivation from the heart using this assay. The presence of viral DNA at 100 dpi has been shown, suggesting long-term maintenance of MCMV within the heart (21). However, the presence of viral DNA does not differentiate between lowlevel replication and establishment of latency in the heart. Thus, the explant reactivation assay was used to evaluate latent MCMV infection and whether MCMV can reactivate from the heart. Following echocardiography at $50 \mathrm{dpi}$, hearts and spleens were cultured ex vivo for 6 weeks to assess MCMV reactivation. Figure 6 demonstrates that both spleens and hearts were latently infected at the time of explant, which is illustrated by the absence of replicating virus at 7 dpe, as determined by plaque assay of culture supernatant. The majority of infected mice (89\%) reactivated virus from both the hearts and spleens. Despite having a pathology score of 1 ; one animal did not reactivate the virus from either tissue. Interestingly, virus reactivation from the heart appeared to occur more readily than from infected spleens. Further studies are required to fully characterize this phenomenon. However, this novel result suggests that latent virus in the heart at $50 \mathrm{dpi}$ is capable of reactivation, which may explain the exacerbation of fibrosis and hypertrophy. Repeated reactivations would likely result in host-induced 


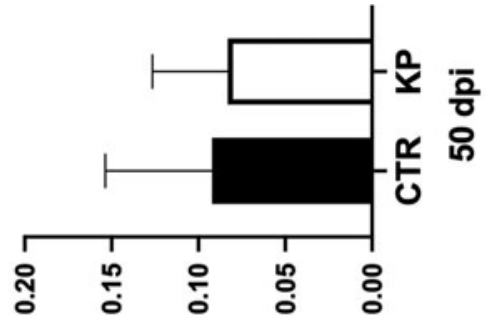

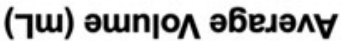

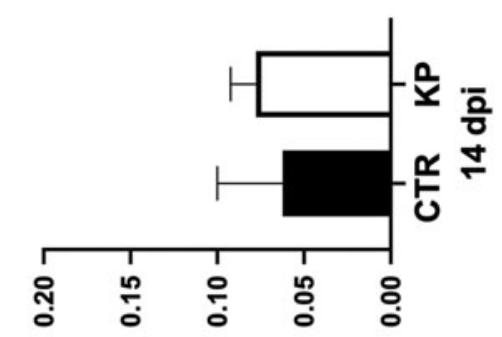

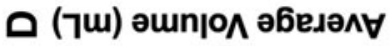
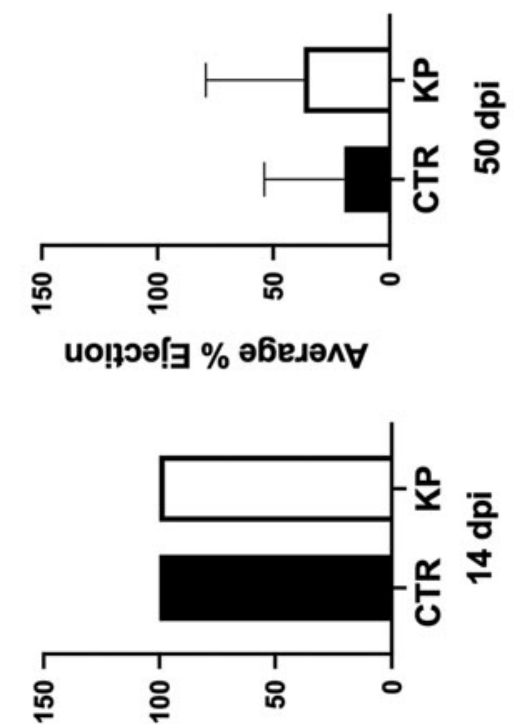

ш ио!ฺออ!ฺ \% әбеมәлท

岳昱
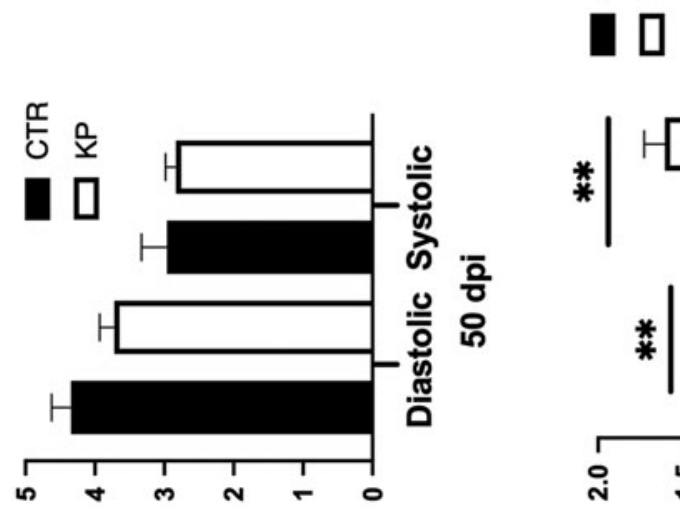

(uس) дәрәше! әбеләл甘

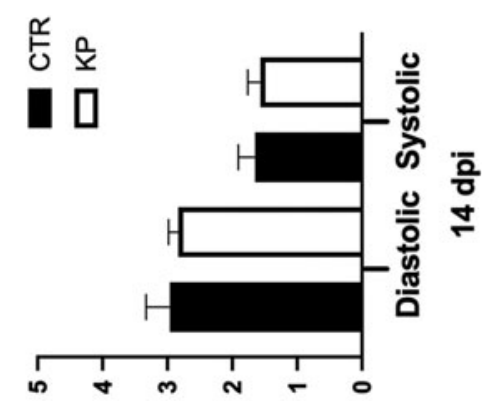

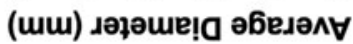
$\varangle$

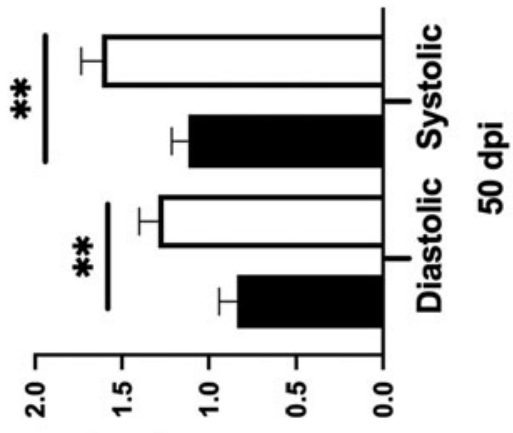

(uiu) ssəuYग!प। ә0snW әбеләл甘

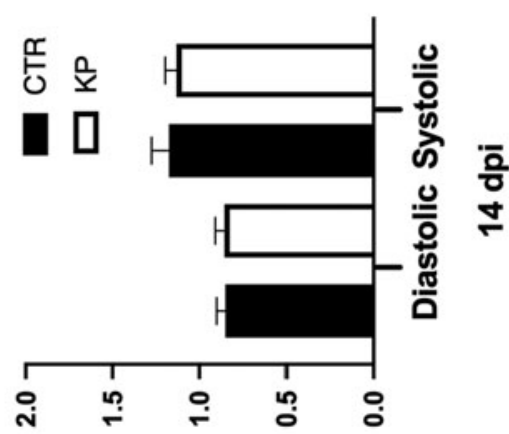

(uu) ssəuxग!บ।

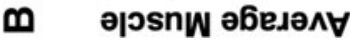

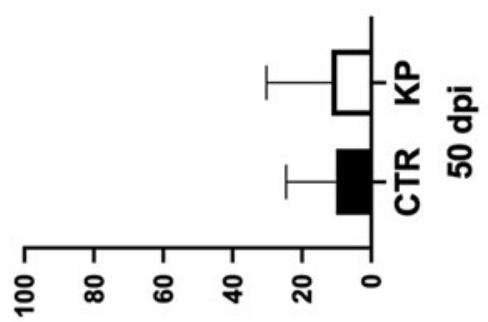

6u!̣วนочs \% әбедәл७

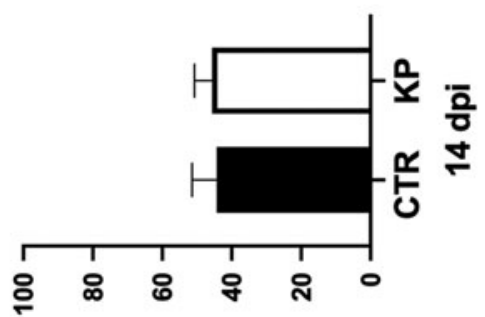

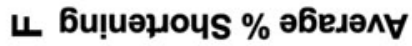

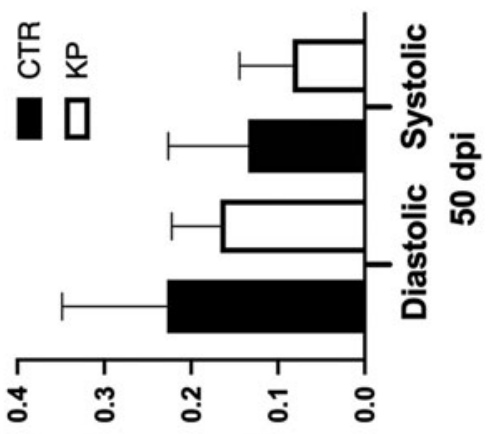

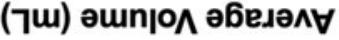

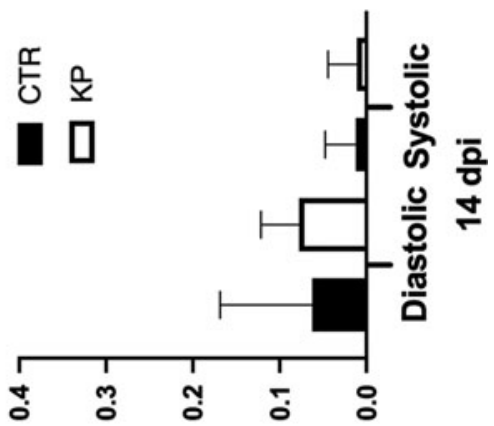

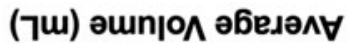

0 


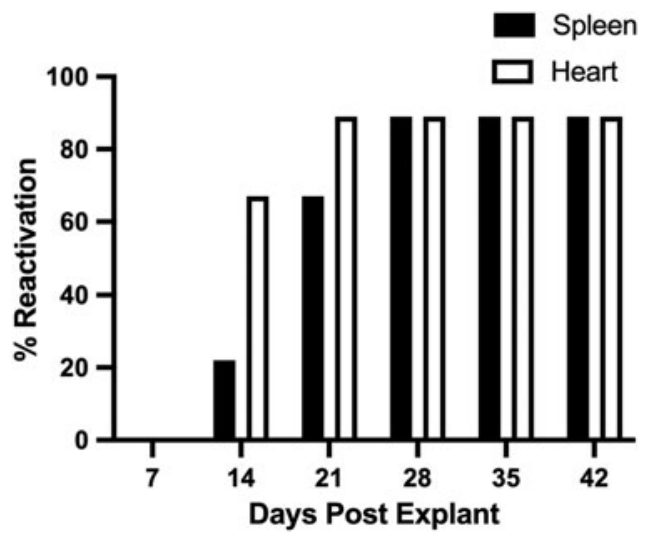

FIG. 6. Ex vivo heart reactivation at 50 dpi. MCMVinfected hearts were cultured ex vivo and evaluated weekly by plaque assay for viral reactivation. The spleen was evaluated as an assay control. Data are presented as percent reactivation per animal. Studies were conducted in triplicate $(n=9)$.

inflammation and wound healing, which is often characterized by collagen accumulation (36), the major cause of fibrosis.

\section{Discussion}

Cytomegalovirus has been associated with cardiovascular dysfunction (34). While viral myocarditis is infrequent in immunocompetent individuals, these cases do occur and can be life-threatening without drug intervention (27). Therefore, understanding the viral mechanisms at play is crucial for improving diagnosis and treatment of future patients. In this article, we have shown that MCMV infection of the heart results in phenotypic alteration and dysfunction that persist during latent infection. MCMV infection in the heart is detectable at $3 \mathrm{dpi}$, peaks at $7 \mathrm{dpi}$, and is below the limit of detection by $14 \mathrm{dpi}$, similar to previous studies $(21,30)$. When comparing the heart with other MCMV-infected tissues, such as the spleen, liver, lungs, and salivary glands, virus replication is relatively low, suggesting that either the infection is limited or the virus does not efficiently replicate in the heart. Very little is known about MCMV infection in the heart and additional studies are required to evaluate which cell types are acutely infected and which cells harbor the latent virus.

Following MCMV infection, white deposits develop on the surface of the heart. This observation has been shown previously; however, the authors identified the visible white deposits in the pericardium as calcification, but no assay confirmed the presence of calcium $(13,30)$. Cardiac calcification has been identified in coxsackievirus B infection of the heart $(12,26,33)$. However, no reports have documented that HCMV or MCMV increased calcium in the heart. The results of this study show accumulation of collagen around the pericardium at $14 \mathrm{dpi}$, as shown by Masson's trichrome staining, which suggests that the deposits are fibrotic. However, additional studies are needed to fully characterize the components of white deposits. Pathology scoring of the white fibrous deposits allowed for a qualitative assessment and demonstrated that infected animals resulted in significantly more deposits compared with uninfected controls.
Surprisingly, the fibrotic pathology score was consistent across both the early and later time points, suggesting that the deposits may be caused by the initial infection and do not appear to worsen over time. HCMV infection has been shown to rearrange the extracellular matrix of fibroblasts and smooth muscle cells in vitro and alter the expression of collagen (29). It is possible a similar mechanism is at play during infection with MCMV.

Interestingly, in addition to white deposits on the heart, MCMV infection resulted in fibrosis of the heart at $14 \mathrm{dpi}$, which was significantly exacerbated at $50 \mathrm{dpi}$. MCMV infection induces fibrosis in a number of tissues (7), including the heart (8), which further supports the observations of this study. However, unlike the white deposits, which did not change, long-term infection resulted in exacerbation of fibrosis. The development from interstitial fibrosis at 14 dpi to replacement fibrosis at 50 dpi suggests that the damage was progressive (8). This phenomenon suggests that MCMV infection may continually stimulate the microenvironment of the tissue, perhaps due to repeated viral reactivation events. A previous study showed that in lungs of MCMVinfected mice, stochastic ie1 expression is enough to induce resident CD8 T cell function (33). Latent MCMV, similar to other herpesviruses, would be expected to occasionally reactivate from latency, thus stimulating repeated MCMVspecific $\mathrm{CD}^{+} \mathrm{T}$ cell responses (33). The hearts were shown to reactivate MCMV by ex vivo culture, suggesting that in vivo reactivation is possible and could result in tissue damage by factors such as cytokine expression, extracellular matrix remodeling, or immune response.

Due to phenotypic alterations observed in the heart, we evaluated whether MCMV infection causes cardiac dysfunction. Echocardiography was used for real-time evaluation of the heart rate, muscle thickness, and rhythm of beating. Our data show that MCMV infection causes increased heart rate at 14 and 50 dpi when compared with uninfected controls. Interestingly, unlike the exacerbation of fibrosis at $50 \mathrm{dpi}$, the heart rate remained consistent between time points. This suggests that the increase in heart rate is associated with damage from the initial infection. Our analysis revealed significant hypertrophy of the left ventricle in MCMV-infected animals at $50 \mathrm{dpi}$, most likely due to fibrosis found in the hearts, which would require the muscle to work harder to maintain the same efficiency as before infection. Hypertrophy is initially compensatory and maintains normal cardiac output; however, long-term distress can result in ventricular overload and heart failure (2). A previous study showed that mice lacking expression of IL-6 within the myocardium were unable to induce muscular hypertrophy and progress directly to cardiomyopathy, suggesting that long-term IL-6 expression could contribute to significant hypertrophy $(2,36)$. Both HCMV and MCMV have been shown to increase IL-6 expression during infection $(30,34)$. When evaluating the rhythm of beating, a momentary, but easily detected, spasm was observed during diastole in many of the infected animals. This suggests that there was a slight lag in the electrical signal sent through the heart, which could result from fibrosis. This correlates with other viral infections in humans, which also cause abnormal ventricular ejection (14). Both heart rate and hypertrophy results shown here mimic HCMV-induced myocarditis $(18,24,27)$. 
Our data show phenotypic and functional alterations, which are exacerbated during latency. We hypothesized that this could be due to frequent MCMV reactivation events, which promote damage. To test this, hearts were cultured ex vivo and evaluated for viral reactivation. In this study, we showed that MCMV is capable of reactivation from the heart, as shown by ex vivo culture. Surprisingly, at 14 dpe, the hearts reactivated the virus with higher frequency than the spleen (1). To further understand MCMV latency in the heart, additional studies are needed to evaluate the cell types harboring latent MCMV as well as viral gene expression during latency within the heart. Repeated viral reactivation would likely result in changes within the microenvironment of the heart due to CD8 T cell responses and chronic cytokine expression such as IL-6, TGF $\beta$, and $\mathrm{TNF} \alpha$, resulting in exacerbated dysfunction of the tissue over time. Similarly, reactivation of HCMV from latently infected transplant hearts increases organ rejection (34).

In conclusion, this article illustrates a high degree of phenotypic and functional deficiencies caused by MCMV infection at 14 and $50 \mathrm{dpi}$, at times when the heart and most other tissues (excluding salivary glands) do not harbor replicating virus. Increased heart rate, hypertrophy, and vascular spasms occur during both HCMV- and MCMVinduced myocarditis. The exacerbated injury at 50 dpi was most likely due to fibrosis, which caused the compensatory hypertrophy to maintain cardiac output and resulted in vascular spasms. Our results indicate that MCMV infection induces changes in the microenvironment of the heart that lead to phenotypic alteration and long-term cardiac dysfunction and may shed insights into HCMV-associated cardiovascular diseases.

\section{Author's Note}

The hot topic at the time was $\mathrm{T}$ cell exhaustion...Peter was interested in studying the $\mathrm{CD} 8^{+} \mathrm{T}$ cell response to a persistent herpesvirus called murine gamma herpesvirus- 68 (MHV-68) and he was looking for a herpes virologist to join his laboratory. My postdoc in the Mocarski laboratory at Stanford University on MCMV pathogenesis and latency provided me with the relevant in vivo herpesvirus training, so I left Palo Alto and moved to Memphis. Herpesviruses, such as cytomegalovirus, herpes simplex virus, or EpsteinBarr virus, employ multiple immune evasion strategies to persist or establish latency for the lifetime of the host. Those early days of MHV-68 research shed new insights into the immunological control of long-term latent infection, including a highly cited article, where we showed that longterm infection was not controlled by $\mathrm{CD}^{+} \mathrm{T}$ cells without the help of $\mathrm{CD}^{+} \mathrm{T}$ cells. My time in Peter's laboratory provided a strong foundation for studying immune mechanisms controlling herpesvirus pathogenesis and latency. It was a privilege to be mentored by Peter, and 1996 was an exciting time in the Doherty laboratory!

\section{Acknowledgment}

The authors would like to thank Sherry Ring for processing and staining of hearts for histology analysis.

\section{Author Disclosure Statement}

No competing financial interests exist.

\section{Funding Information}

This work was supported by the National Institutes of Health grant R01 AI087683-01A1, the LSU School of Veterinary Medicine Startup Fund to R.D.C., and an LSU Economic Development Assistantship to C.M.B.

\section{References}

1. Almanan M, Raynor J, Sholl A, et al. Tissue-specific control of latent CMV reactivation by regulatory $\mathrm{T}$ cells. PLoS Pathog 2017;13:8.

2. Barry S, Townsend P, Latchman D, and Stephanou A. Role of the Jak-Stat pathway in myocardial injury. Trends Mol Med 2006;13:82-89.

3. Borthwick L, Wynn T, and Fisher A. Cytokine mediated tissue fibrosis. Biochim Biophys Acta 2013;1832:1049-1060.

4. Cardin RD, Schaefer G, Allen J, Davis-Poynter N, and Farrell H. The M33 chemokine receptor homolog of murine cytomegalovirus exhibits a differential tissue-specific role during in vivo replication and latency. J Virol 2009;83: 7590-7601.

5. Caruso A, Rotola A, Comar M, et al. HHV-6 infects human aortic and heart microvascular endothelial cells, increasing their ability to secrete proinflammatory chemokines. J Med Virol 2002;67:528-533.

6. Cheng J, Ke Q, Wang H, et al. Cytomegalovirus infection causes an increase of arterial blood pressure. PLoS Pathog 2009;5:1-14.

7. Clement M, and Humphreys I. Cytokine-mediated induction and regulation of tissue damage during cytomegalovirus infection. Front Immunol 2019;10:1-5.

8. Cook C, Bickerstaff A, Wang J, et al. Disruption of murine cardiac allograft acceptance by latent cytomegalovirus: disruption of murine cardiac allograft. Am J Transplant 2008;9:42-53.

9. Dange R, Agarwal D, Masson G, et al. Central blockade of TLR4 improves cardiac function and attenuates myocardial inflammation in angiotensin 2-induced hypertension. Cardiovasc Res 2014;103:17-27.

10. Dumortier J, Streblow D, Moses A, et al. Human cytomegalovirus secretome contains factors that induce angiogenesis and wound healing. J Virol 2008;82:65246535 .

11. Fields BN, Knipe DM, Howley PM, and Griffin DE. Fields virology. Philadelphia: Lippincott Williams \& Wilkins, 2001.

12. Funseth E, Lindh U, Wesslen L, Friman G, and Ilback $\mathrm{N}$. Trace element changes in the myocardium during Coxsackievirus B3 myocarditis in the mouse. Biol Trace Elem Res 2000;76:149-160.

13. Gang D, Barrett L, Wilson E, Rubin R, and Medearis D. Myopericarditis and enhance dystrophic cardiac calcification in murine cytomegalovirus infection. J Am Pathol 1986;124:207-215.

14. Gavin J, Maxwell L, and Edgar S. Microvascular involvement in cardiac pathology. J Mol Call Cardiol 1998;30: 2531-2540.

15. Jacobs F, Knoop C, Brancart F, et al. Human Herpesvirus-6 infection after lung and heart-lung transplantation: a prospective longitudinal study. Transplantation 2003;75:19962001.

16. Kuhl U, Lassner D, Wallaschek N, et al. Chromosomally integrated human herpesvirus 6 in heart failure: prevalence and treatment: prevalence and reactivation of ciHHV-6. Eur J Heart Failure 2015;17:9-19. 
17. Kuhl U, Pauschinger M, Noutsias M, et al. High prevalence of viral genomes and multiple viral infections in the myocardium of adults with "idiopathic" left ventricular dysfunction. Circulation 2005;111:887-893.

18. Kyto V, Vuorinen T, Saukko P, et al. Cytomegalovirus infection of the heart is common in patients with fatal myocarditis. Clin Infect Dis 2005;40:683-688.

19. Lautenschlager I, and Razonable R. Human herpesvirus-6 infection in kidney, liver, lung, and heart transplantation: review. Transplant Int 2012;25:493-502.

20. Lee S, Brook E, Affandi J, et al. A high burden of cytomegalovirus marks poor vascular health in transplant recipients more clearly than in the general population. Clin Transl Immunol 2019;8:1-8.

21. Lenzo J, Fairweather D, Cull V, Shellam G, and Lawson C. Characterisation of murine cytomegalovirus myocarditis: cellular infiltration of the heart and virus persistence. J Mol Cell Cardiol 2002;34:629-640.

22. Lenzo J, Shellam G, and Lawson C. Ganciclovir and cidofovir treatment of cytomegalovirus-induced myocarditis in mice. Antimicrob Agents Chemother 2001;45:1444 1449.

23. Leung W, Hata J, and Hashimoto K. Murine cytomegalovirus infection model in balb/c mice-pericarditis with myocardial involvement during virus infection. Tokai J Exp Clin Med 1986;11:303-311.

24. Magno Palmeira M, Ribeiro H, Lira Y, et al. Heart failure due to cytomegalovirus myocarditis in immunocompetent young adults: a case report. BMC Res Notes 2016;1:391.

25. Oh J. Echocardiography in heart failure: beyond diagnosis. Eur J Echocardiogr 2007;8:4-14.

26. Oka K. Oohira K, Yatabe Y, et al. Fulminant myocarditis demonstrating uncommon morphology-a report of two autopsy cases. Virchows Arch 2005;446:259-264.

27. Padala S, Kumar A, and Padala S. Fulminant cytomegalovirus myocarditis in an immunocompetent host: resolution with oral valganciclovir. Tex Heart Inst J 2014;41:523-529.

28. Rai V, Sharma P, Agrawal S, and Agrawal D. Relevance of mouse models of fibrosis and hypertrophy in cardiac research. Mol Cell Biochem 2017;424:123-145.

29. Reinhardt B, Winkler M, Schaarschmidt P, et al. Human cytomegalovirus-induced reduction of extracellular matrix proteins in vascular smooth muscle cell cultures: a pathomechanism in vasculopathies? J Gen Virol 2006;87:28492858.

30. Ritter J, Tang-Feldman Y, Lochhead R, et al. In vivo characterization of cytokine profiles and viral load during murine cytomegalovirus-induced acute myocarditis. Cardiovasc Pathol 2010;19:83-93.
31. Schonian U, Crombach M, and Maisch B. Assessment of cytomegalovirus DNA and protein expression in patients with myocarditis. Clin Immunol Immunopathol 1993;68: 229-233.

32. Seki A, and Fishbein M. Predicting the development of cardiac allograft vasculopathy. Cardiovasc Pathol 2014;23: 253-260.

33. Simon C, Holtappels R, Tervo $\mathrm{H}$, et al. CD8 T cells control cytomegalovirus latency by epitope-specific sensing of transcriptional reactivation. J Virol 2006;80:10436-10456.

34. Soderberg-Naucler C. Does cytomegalovirus play a causative role in the development of various inflammatory diseases and cancer? J Int Med 2006;259:219-246.

35. Stoddart C, Cardin R, Boname J, et al. Peripheral blood mononuclear phagocytes mediate dissemination of murine cytomegalovirus. J Virol 1994;68:6243-6253.

36. Suthahar N, Meijers W, Sillje H, and de Boer R. From inflammation to fibrosis-molecular and cellular mechanism of myocardial tissue remodeling and perspectives on differential treatment opportunities. Curr Heart Fail Rep 2017; 14:235-250.

37. Tabata T, Kawakatsu H, Maidji E, et al. Induction of an epithelial integrin avb6 in human cytomegalovirus-infected endothelial cells lead to activation of transforming growth factor-b1 and increased collagen production. Am J Pathol 2008;172:1127-1138.

38. Vleigen I, Duijvestijn A, Grauls G, et al. Cytomegalovirus infection aggravates atherogenesis in apoE knockout mice by both local and systemic immune activation. Microbes Infect 2004;6:17-24.

39. Vleigen I, Stassen F, Grauls G, Blok R, and Bruggeman C. MCMV infection increases early T-lymphocyte influx in atherosclerotic lesions in apoE knockout mice. J Clin Virol 2002;25:159-171.

40. Wall N, Chue C, Edwards N, et al. Cytomegalovirus seropositivity is associated with increased arterial stiffness in patients with chronic kidney disease. PLoS One 2013;8:1-7.

41. Weill D. Role of cytomegalovirus in cardiac allograft vasculopathy. Transplant Infect Dis 2001;3:44-48.

Address correspondence to: Dr. Rhonda D. Cardin Department of Pathobiological Sciences School of Veterinary Medicine

Louisiana State University

Baton Rouge, LA 70803-2804

E-mail: rcardin@1su.edu 